\title{
Mixture Perception of rORI7 Agonists with Similar Odors
}

\author{
Anne J. Kurtz • John Barnard • Terry E. Acree
}

Received: 16 September 2010 / Accepted: 15 August 2011 /Published online: 3 September 2011

(C) Springer Science+Business Media, LLC 2011

\begin{abstract}
The straight-chain aldehyde hexanal has a distinct "green-grassy" smell quite different from the similar "citruswaxy"-smelling homologues heptanal to undecanal (Kittel et al., Chemosens Percept 1:235-241, 2008). Two prior studies demonstrated the absence of cross-adaptation between hexanal and three other homologues (Kurtz et al., Chemosens Percept 3:149-155, 2010) but a significant mixture suppression between the dissimilar-smelling odorants hexanal and octanal (Kurtz et al., Chemosens Percept 4:186-194, 2009). In contrast, this study of similar-smelling octanal and decanal showed little mixture suppression. In contrast to the hexanal and octanal adaptation, data from Kurtz et al. (Chemosens Percept 3:149-155, 2010) showed a significant cross-adaptation between octanal and decanal. The differences observed between mixture suppression and adaptation suggest that these two phenomena are processed separately.
\end{abstract}

Keywords Olfaction - Synthetic perception - Analytic perception · Matching task - Dose-response curves - Mixture suppression $\cdot$ Cross adaptation

\section{Introduction}

Background

In the emerging view of flavor perception, humans are able to experience complex mixtures of stimuli in two different ways: analytically (elemental processing or bottom-up) and synthetically (configural processing or top-down). Not only can humans be trained to do this in controlled experimental

\footnotetext{
A. J. Kurtz · J. Barnard · T. E. Acree $(\bowtie)$

Food Science, Cornell University,

Geneva, NY 14456, USA

e-mail: tea2@cornell.edu
}

settings (Prescott 2004; Gottfried 2010; Small and Prescott 2005), but this is also a natural human process. It is not unusual to hear a statement such as, "The odor of this wine has a weak Riesling character (synthesis) and a strong citrus note (analysis)," indicating these notions apply within a single sensory modality such as olfaction (Prescott 2004). It has been proposed that analytical processing results from attention to the component sensations arising at the periphery and synthetic observations derived from the multimodal integration of these sensations in the central nervous system (Auvray and Spence 2008). If true, then knowledge of the psychophysics of single odorants in mixtures may relate to the neurobiology and anatomy of the olfactory system at the periphery of the central nervous system. This paper investigates the ability of subjects to attend to individual odorants in a mixture using an analytical protocol based on intensity matching instead of perceptual scaling (Kurtz et al. 2010).

In olfactory "analysis," subjects are asked to attend to a component of a mixture of odorants and to evaluate its intensity separately from the intensity of the whole sample. But measuring sensory "quality" of any kind is challenging; obviously, there is no way to know or measure directly a subject's experience (Gottfried 2010). Only a subject's behavior in response to a stimulant can be measured directly. However, clever experiments can measure perceptions indirectly: Among the most ingenious is Ramachandran's use of response time to distinguish subjects who experience color-number associations from those who do not (Ramachandran and Hubbard 2001).

A single odorant above its threshold usually produces a weaker perception in a mixture than it does when smelled alone. Called mixture suppression, this phenomenon may also be responsible for our inability to easily detect more than three odorants in a mixture (Cain and Drexler 1974; Köster 1968; Berglund and Olsson 1993; Goyert et al. 2007; Jones and 
Woskow 1964; Laing et al. 1984; Brossard et al. 2007). In contrast, mixtures of two or three odorants prepared at perithreshold concentrations show indication of enhancement (Small and Prescott 2005; Delwiche 2004; Diamond et al. 2005; Veldhuizen et al. 2010; Cometto-Muniz et al. 2007; Miyazawa et al. 2009). Clearly, odorants function differently near their threshold than at higher levels. To minimize perithreshold effects, an effort was made in the experiments discussed here to use stimulus levels that were suprathreshold, distinguishable from each other, and covering much of the dynamic range of their intensities.

A measurement based on olfactory "synthesis" directs the subject to estimate the intensity of the overall olfactory experience, a process that has yielded reproducible quantifications especially within a single laboratory (Nagata 2002; Cain and Drexler 1974). Studies of mixture perception using "synthetic" protocols have also yielded theories, e.g., the vector theory of binary odor perception, that predict the intensity of mixtures from the intensity of their components tested separately (Berglund et al. 1973). In the seminal work of Berglund et al. (1973), subjects were trained to scale their perceptions of odor mixture intensity as a single response (synthetic) using external standards not present in the test mixture as references (Berglund et al. 1973). This increased the probability that subjects used a synthetic strategy to scale the intensity of the mixture since the reference did not focus the subject's attention on a single component within the mixture.

In contrast, Cain and Drexler (1974) and Köster (1969) trained their subjects to analyze mixtures, i.e., to attend to and scale a component odor separate from the whole, but still used an external reference. We theorized that by training subjects to match a reference of the same stimulus as the test odorant would focus their attention on the analyte and produce more "analytical" results. Although we do not examine this theory experimentally in this study, the approach seems unlikely to produce less analytic data than the use of an external standard.

This is the fourth in a series of papers (Kittel et al. 2008; Kurtz et al. 2009, 2010) examining the human psychophysics of the primary agonists of the rat olfactory receptor ORI7, the first mammalian deorphanized olfactory receptor (Zhao et al. 1998). The primary agonists of rORI7 are the normal aldehydes $\mathrm{C} 7-\mathrm{C} 11$ and have a similar "citrus-like" odor quality for humans. The homologue $\mathrm{C} 6$ has been hypothesized to act as antagonist for rORI7 and has a distinct "green/grassy" smell (Peterlin et al. 2008) (Kittel et al. 2008). Our second paper demonstrated cross-adaptation in humans between the rORI7 agonists (C7-C11), while antagonist hexanal (C6) did not cross-adapt with C7-C11. The final two experiments examined the perceived intensity of three of these compounds, C6, C8, and C10, in binary mixtures using an analytical protocol that uses reference matching instead of scaling (Kurtz et al. 2010, 2009). Other studies (Köster 1968; Berglund et al. 1973; Cain and Drexler 1974; Laing and Francis 1989) show reproducible patterns of odor processing of mixtures from the psychophysics of single odorants that are not the same for all odorants in mixtures. Our examination of hexanal (C6) and octanal (C8) in the same protocols used in this paper for C8 and $\mathrm{C} 10$ demonstrated no cross-adaptation in experiments with $\mathrm{C} 8$ and $\mathrm{C} 6$ and subjects experienced mixture suppression (Kurtz et al. 2010). This paper adds to the previous studies by analyzing the effect of compounds that exhibit cross-adaptation (C8 and $\mathrm{C} 10)$.

\section{Methods}

Subjects

Nine female and two male, nonsmokers with olfactory function and a mean age of $26.3 \pm 1.5$ years, volunteered to participate. The research protocol was reviewed and approved by the University Committee on Human Subjects of Cornell University. All subjects were paid for their participation.

\section{Materials}

Odorants octanal 99\% (C8) and decanal 99\% (C10) were obtained from Sigma-Aldrich (St. Louis, MO, USA). All odorants were reagent grade and dissolved in odorless polyethylene glycol (PEG) 400 from J.T. Baker (Phillipsburg, NJ, USA). Nine concentrations were tested during the threealternative forced choice dose-response curve collection. The lowest concentration contained only pure PEG with no odor added. Concentrations for $\mathrm{C}_{8}$ ranged from 0.44 to $965 \mathrm{nM}$ by factors of 3. Concentrations for $\mathrm{C}_{10}$ ranged from $9.8 \times 10^{-3}$ to $21.6 \mu \mathrm{M}$.

All odorants were presented in $250-\mathrm{mL}$ polyethylene VWR wash bottles, modified with a $1.5-\mathrm{cm}$ diameter Teflon ball fitted around the neck of the bottle for nasal comfort and at room temperature in a well-ventilated space. Bottles were labeled with a random three-digit code and prepared $1 \mathrm{~h}$ prior to testing. Each bottle contained two blotters. For bottles containing "no odor," two strips of pure PEG were placed into the bottle. Bottles that held odorants contained one strip dipped in odorant and another dipped in pure PEG.

\section{Dose-Response Curve Collection}

Dose-response curves were collected in order to determine the three concentration levels for each compound to be used 
in the reference matching task. At the beginning of each session, subjects were presented with two references, one contained pure PEG, while the other contained the highest concentration of the test odorant. Subjects were instructed to familiarize themselves with the target odorant. The odorant reference given to each subject corresponded with the odorant tested. Individuals were presented with a series comprised of nine groups in each session. Each concentration step was assigned a label A through I. Step A contained only pure PEG, and no target odorant; its purpose was to determine the level of noise contributed by the solvent, PEG, and the "plastic" odor of the polyethylene squeeze bottles. Each step consisted of nine bottles, arranged in rows of three. Each subject evaluated all nine groups in one session. The process was repeated two times for each odor, in separate sessions. Each row represented a trial. As in the method described by Wise and Cain (2000), the individual evaluated three bottles, searching for the bottle with the target odorant (Wise and Cain 2000). For all steps except step A, two of the bottles contained blanks (PEG), while one bottle contained the target odorant (in step A, all three bottles contain PEG). The subject recorded information including: bottle number (the three-digit code on the bottle), bottle location, perceived intensity, and level of confidence in his or her choices. The subject used a ninepoint scale to evaluate perceived intensity $(1=$ no odor, $9=$ very strong). Between each evaluation, the subject rested during a timed 45 -s break. If during testing an individual expressed difficulty perceiving odors, the subject took a timed 5-min break. Each test session took approximately $45 \mathrm{~min}$. Every subject completed two sessions per odorant tested, for a total of four sessions. The test order, whether a subject received $\mathrm{C} 8$ or $\mathrm{C} 10$ to test, was randomized across sessions. The subjects received no prior training but were experienced members of other sensory experiments.

Stimulus Levels Used in the Odor Matching Task

Based on the results from the dose-response curves, three concentration levels were determined for both $\mathrm{C} 8$ and $\mathrm{C} 10$ representing: low $(\mathrm{L})$, medium $(\mathrm{M})$, and high $(\mathrm{H})$ as seen in Figs. 1 and 2. In addition to the three $\mathrm{L}, \mathrm{M}$, and $\mathrm{H}$ references used in Kurtz et al. 2009, a fourth reference containing only PEG (Ø) was added. Concentration levels defined as $\mathrm{L}, \mathrm{M}$, and $\mathrm{H}$ were chosen to be clearly suprathreshold ( $>5 \%$ detection), statistically distinguishable, and to cover most of the range of the intensity scores given. The solutions used to collect the dose-response curves were also used during the reference matching task. Bottles containing binary mixtures contained two blotters: one blotter dipped in $\mathrm{C} 8$ and another in C10. A mixture written as $8 \mathrm{~L}-10 \mathrm{M}$ contains a low concentration of $\mathrm{C} 8$ and a medium concentration of $\mathrm{C} 10$. There were five binary mixture stimuli: $8 \mathrm{M}-10 \mathrm{~L}, 8 \mathrm{M}-10 \mathrm{M}, 8 \mathrm{M}-10 \mathrm{H}, 8 \mathrm{~L}-10 \mathrm{M}$, and $8 \mathrm{H}-10 \mathrm{M}$. All stimuli were prepared using the same procedure outlined in Kurtz et al. (2009).

\section{Subject Training for Odor Matching Task}

As in Kurtz et al. (2009), subjects were trained to distinguish four concentrations: $\varnothing, \mathrm{L}, \mathrm{M}$, and $\mathrm{H}$ for both $\mathrm{C} 8$ and $\mathrm{C} 10$. Although 11 subjects were trained, one female subject was unable to participate in the final experiment. As in Kurtz et al. (2009), training consisted of individuals matching several bottles made up of $\varnothing, \mathrm{L}, \mathrm{M}$, and $\mathrm{H}$ concentrations to corresponding references of $\varnothing, \mathrm{L}, \mathrm{M}$, and $\mathrm{H}$.
Fig. 1 Dose response curve for C8. Participants received eight different concentrations. Log (nM) concentrations of $\mathrm{C} 8$ are plotted versus mean perceived intensity (black dots), their LSIs based on pooled error from the ANOVA (whiskers) and mean percent correct bottle identification (open circles). Points marked Low, Medium, and High were the concentration levels chosen for the references

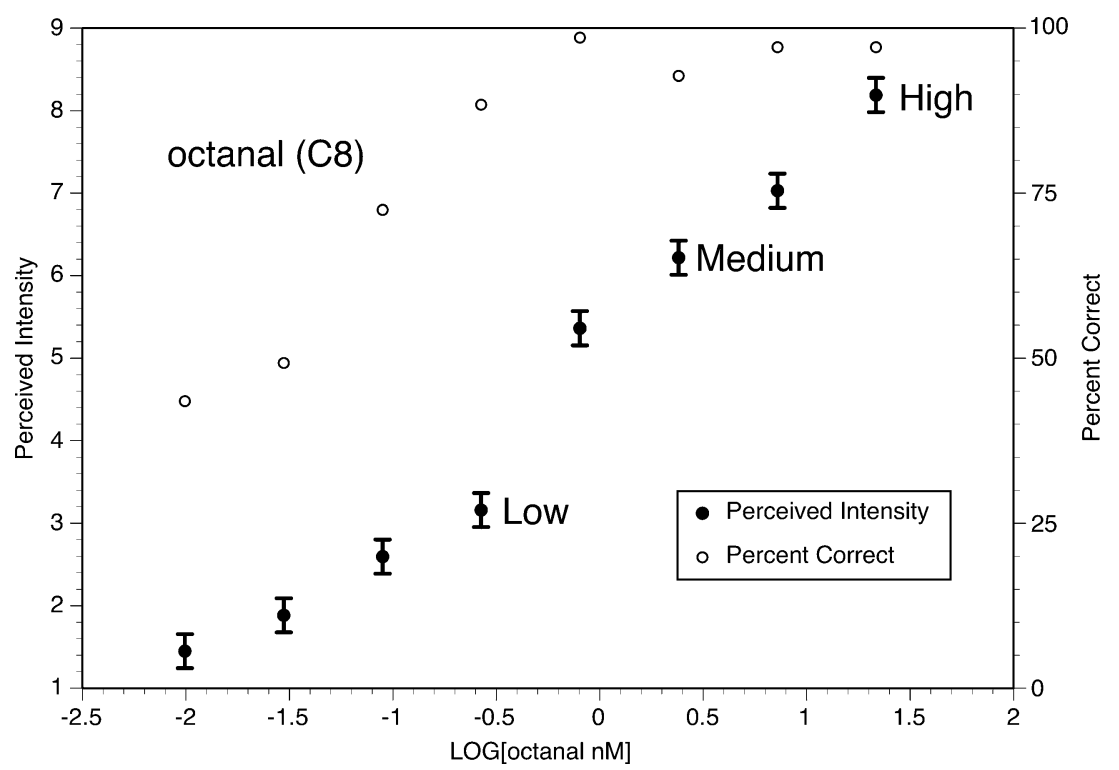


Fig. 2 Dose response curve for C10. Participants received eight different concentrations. Log $(\mu \mathrm{M})$ concentrations of $\mathrm{C} 8$ are plotted versus mean perceived intensity (black dots), their LSIs based on pooled error from the ANOVA (whiskers) and mean percent correct bottle identification (open circles). Points marked Low, Medium, and High were the concentration levels chosen for the references

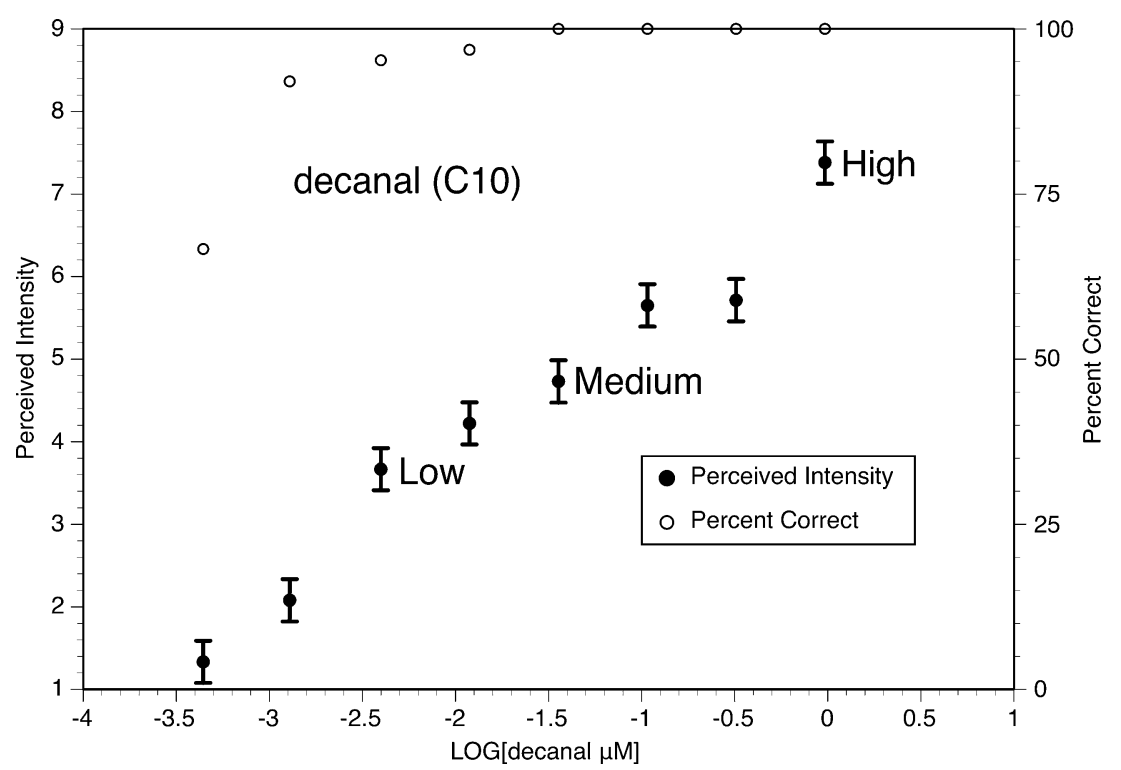

An ABC-X task was used in this procedure. The method resembles an $\mathrm{AB}-\mathrm{X}$ task, in which an individual is required to determine whether $\mathrm{X}$ is more similar to reference $\mathrm{A}$ or reference B (Lawless and Heymann 1999). In an ABC-X task, however, $\mathrm{X}$ (the stimulus in question) can match either to references A, B, or C. Training followed the protocol outlined in Kurtz et al. (2009); however, the ABC-X task was modified to include a reference bottle containing only PEG, marked as $\varnothing$. Odorants $\mathrm{C} 8$ and $\mathrm{C} 10$ were tested separately. During each training session, a subject received nine bottles: two $\varnothing$ bottles, two L bottles, two M bottles, and two $\mathrm{H}$ bottles. An additional bottle of either $\varnothing, \mathrm{L}, \mathrm{M}$, or $\mathrm{H}$ was added as the ninth bottle to ensure that subjects did not use a process of elimination to place all of the bottles. As previously described, each subject sniffed the four references until he or she said the odors were familiar; next, each chose a bottle from the randomly placed set of nine bottles. The subject sniffed the bottle and decided which reference it best matched. He or she then placed the bottle in front of the corresponding reference and rested for $45 \mathrm{~s}$ during a timed break. The process was repeated twice for each odor. In order for a subject to be considered for testing, he or she had to demonstrate $90 \%$ correct bottle placement in the second trial.

\section{Odor Matching Task Procedure}

Subjects were presented with the same four references used in the training session: $\varnothing \mathrm{L}, \mathrm{M}$, and $\mathrm{H}$. As in the training session, subjects were required to match a randomly placed set of bottles in front of the reference which best matched the odorant concentration within the bottle. However, six of the bottles were binary mixtures and three of the bottles were controls. Controls were bottles containing single odorants of the same concentration as the references. Two replicates of nine bottles were presented during each testing session, with a 5-min break in between. As in the training sessions, a 45-s timed break took place between each bottle evaluation.

In separate testing sessions, subjects evaluated the intensities of $\mathrm{C} 8$ and $\mathrm{C} 10$. If the reference bottles contained $\mathrm{C} 8$, the individual would evaluate all test bottles for $\mathrm{C} 8$. If the reference bottles contained $\mathrm{C} 10$, the subject would evaluate all bottles for $\mathrm{C} 10$.

During testing, one odor attended to in a mixture (the figure) was associated with a pure reference of similar intensity and the other (the ground) was to be ignored. It is impossible to know whether an individual could fully ignore the other odorant within the mixture; however, the proper placement of the controls ensures that the individual is capable of properly matching a target to a reference. If the reference contained $\mathrm{C} 8$, the task of the subject was to identify the intensity of $\mathrm{C} 8$, try to ignore the intensity of $\mathrm{C} 10$, and match the bottle to the reference with a similar intensity. One might expect that for a C8 matching task, if an individual were presented with a bottle containing $8 \mathrm{M}-$ $10 \mathrm{~L}$, the subject would identify the medium concentration of $\mathrm{C} 8$, and match it to the $8 \mathrm{M}$ reference. Should the subject experience enhancement or suppression, however, they would place the bottle containing $8 \mathrm{M}-10 \mathrm{~L}$ in front of one of the other references. The average bottle placement across replications and subjects should reflect the degree to which subjects experience enhancement or suppression.

A diagram of the experimental design is available in Kurtz et al. (2009). Two different experiments were conducted: figure varied (FV) and figure constant (FC). Each task was presented when $\mathrm{C} 8$ was the figure and $\mathrm{C} 10$ was the figure; this means there were four different tests $\mathrm{C} 8$ 
FV, C8 FC, C10 FV, and C10 FC. The subjects were presented with tests in randomized order. In the figure varied experiment, binary mixtures with increasing figure intensity were presented, while the ground concentration remained constant; in a $\mathrm{C} 8 \mathrm{FV}$ task, the binary mixtures would be as follows: $8 \mathrm{~L}-10 \mathrm{M}, 8 \mathrm{M}-10 \mathrm{M}$, and $8 \mathrm{H}-10 \mathrm{M}$. In the FC task, the ground concentrations increased, while the figure concentration remained constant at $\mathrm{M}$. The binary mixtures presented in a C8 FC task were: $8 \mathrm{M}-10 \mathrm{~L}, 8 \mathrm{M}-$ $10 \mathrm{M}$, and $8 \mathrm{M}-10 \mathrm{H}$. For $\mathrm{C} 10$, the binary mixtures presented for the FV task were: $10 \mathrm{~L}-8 \mathrm{M}, 10 \mathrm{M}-8 \mathrm{M}$, and $10 \mathrm{H}-8 \mathrm{M}$. The binary mixtures presented in the $\mathrm{C} 10 \mathrm{FC}$ task were: $10 \mathrm{M}-8 \mathrm{~L}, 10 \mathrm{M}-8 \mathrm{M}$, and $10 \mathrm{M}-8 \mathrm{H}$.

If the individual was capable of matching the intensity of the figure odorant to the appropriate reference without any interference from the ground odor in the FC task, he would match all binary mixtures to the appropriate reference. If he were to experience suppression or enhancement, however, he would make the match less frequently.

\section{Statistical Analysis}

Mean perceived intensity and associated uncertainty intervals were calculated for each odorant concentration. The mean of group A (PEG only) was subtracted from the means of groups of B-I, in order to remove "noise." Additionally, percent correct response was calculated for each group. Data for C8 and C10 were analyzed separately. Figures 1, 2, 3, 4, and 5 incorporate significance tests of differences between means using the uncertainty intervals of Andrews et al. (1980). $\alpha=5 \%$ least significant intervals (LSI) were calculated as

$L=\bar{x} \pm \frac{t_{a / 2, v} \sqrt{2 s^{2} / n}}{2}$

where $\bar{x}$ is a mean, $t_{a / 2, v}$ is the upper $\alpha / 2$ point of Student's $t$ distribution on $\nu$ degrees of freedom, $s^{2}$ is the error mean square from analysis of variance, and $n$ is the number of observations contributing to each mean (Andrews et al. 1980). Since least significant intervals include the error mean square from analysis of variance, they are more conservative than confidence intervals. Two means significantly differ if their least significant intervals do not overlap. Only intensity data from trials in which the threeAFC was correct were used to calculate intensity values for Figs. 1 and 2.

\section{Analysis of Binary Mixture Data}

As in Kurtz et al. (2009), data were split into two separate groups. One group was defined as FC, and the other was defined as FV. Each group contained data from both C8 and C10 matching tasks.

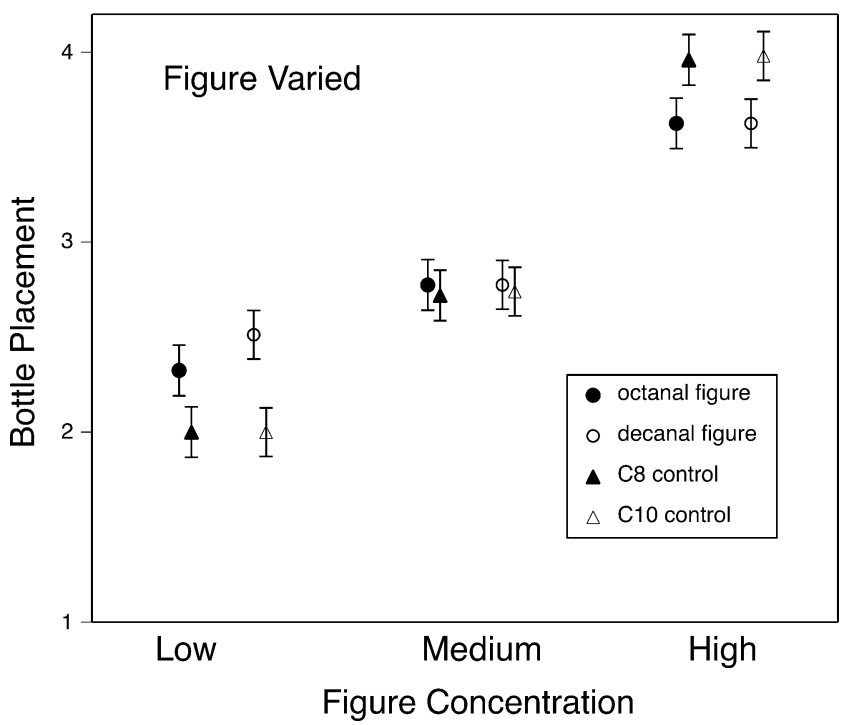

Fig. 3 "Figure varied" compares the mean bottle placement in the odor-intensity matching task (dots) at different figure concentrations but the same Medium level ground concentration to the mean placement of an unmixed figure reference (triangles). Filled points indicate $\mathrm{C} 8$ was the figure, open points indicate when $\mathrm{C} 10$ was the figure and the whiskers indicate the $95 \%$ LSIs based on pooled error from the ANOVA

Means and confidence intervals of bottle placement for each binary mixture were tabulated. Bottle placement was scored accordingly: $\varnothing=1, L=2, M=3$, and $H=4$. Each bottle placement was assigned a score accordingly. Mean bottle placement scores were calculated for all controls and binary mixtures as well as their corresponding LSIs.

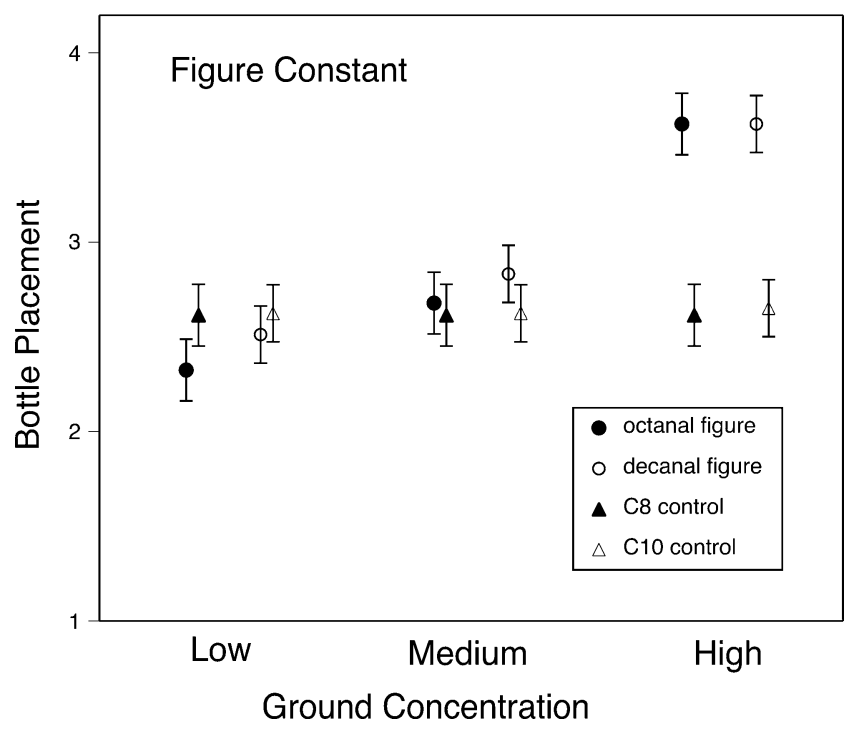

Fig. 4 "Figured constant" compares the mean bottle placement in the odor-intensity matching task (dots) the Medium level of the figure in the presence of different levels of ground to the mean placement of an unmixed figure reference (triangles). Filled points indicate $\mathrm{C} 8$ was the figure, open points indicate when 2 of $3 \mathrm{C} 10$ was the figure and the whiskers indicate the $95 \%$ LSIs based on pooled error from the ANOVA 

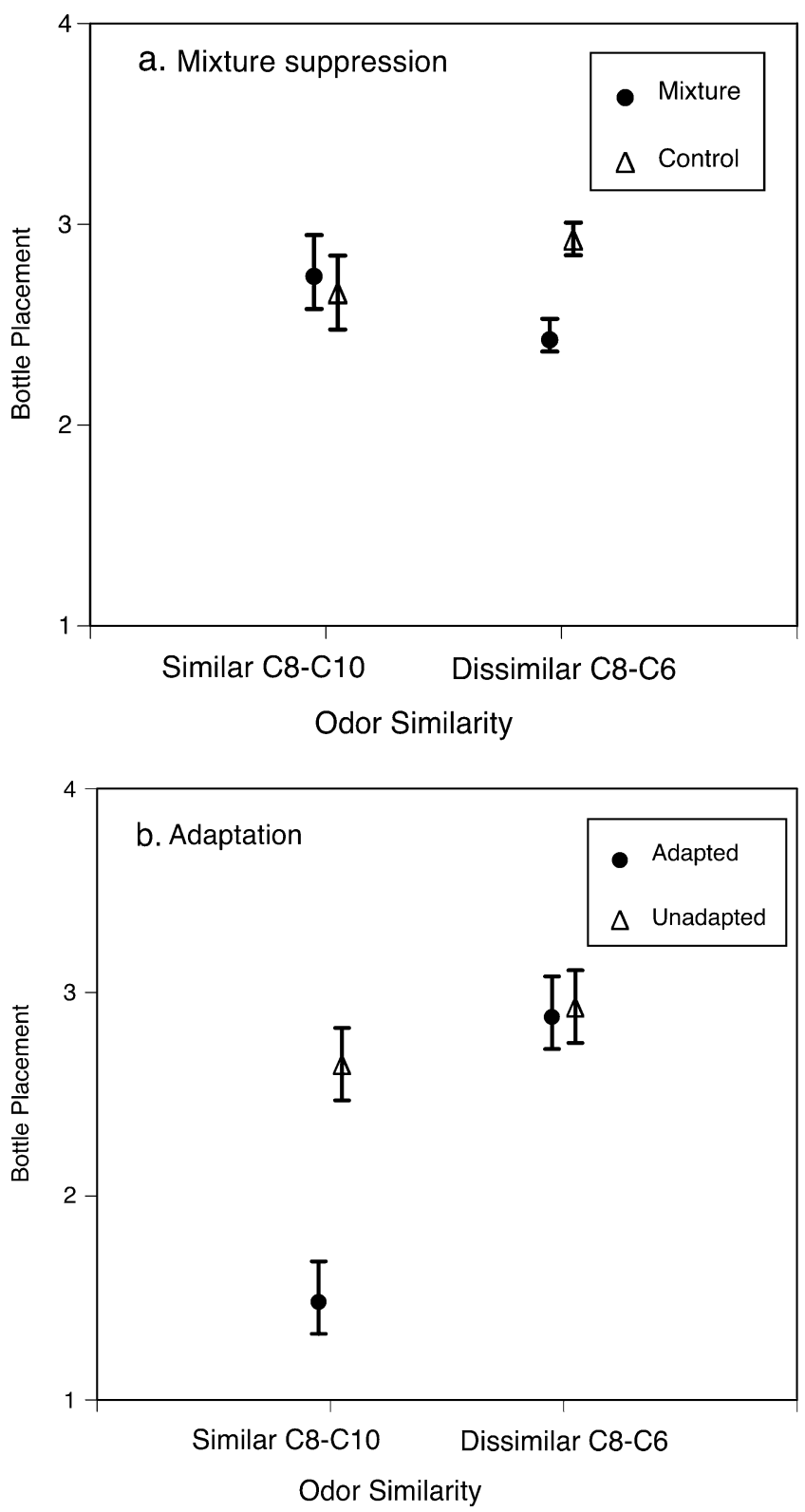

Fig. 5 a 'Mixture Perception' compares mean data of similar smelling $8 \mathrm{M}-10 \mathrm{M}$ mixtures to mean data of the dissimilar smelling $8 \mathrm{M}-6 \mathrm{M}$ (Kurtz et al 2009). Mean bottle placement scores for the mixtures are the black dots and the whiskers indicate the 95\% LSIs based on pooled error from the ANOVA. The open triangles are the mean scores of the unmixed reference. b 'Adaptation' plots data from Kurtz et al (2010), where mean post-adaptation scores (black dots) from the cross adaptation of $\mathrm{C} 8-\mathrm{C} 10$ and $\mathrm{C} 8-\mathrm{C} 6$ are compared to the un-adapted scores open triangles

\section{Results}

Figures 1 and 2 summarize the dose-response data for $\mathrm{C} 8$ and C10: plots of log concentration versus mean perceived intensity and percent correct identification of the odorantcontaining bottle. For both $\mathrm{C} 8$ and $\mathrm{C} 10$, the concentrations chosen span more than $50 \%$ of the intensity scale, significantly differ from each other $(P<0.05)$, and were selected correctly by the subjects more than $80 \%$ of the time. That the corresponding high, medium, and low concentrations were not exactly isointense for $\mathrm{C} 8$ and $\mathrm{C} 10$ was not relevant since the final matching task involved no perceptual scaling. The subjects simply compared the intensity of a mixture to the intensity of three references. The resulting low, medium, and high levels for octanal used in the matching tasks below were 3.9, 35.7, and $965.7 \mathrm{nM}$, respectively, and for decanal, 0.3, 2.4, and 21.6 $\mathrm{MM}$, respectively.

Figures 3 and 4 demonstrate an overall increase in matching score as the figure concentration increases (Fig. 3) or as the ground increases (Fig. 4). As expected, the placement of the low, medium, and high figure controls shown in Fig. 3 (where the ground is constant and always at the medium level) shows homologously increasing values. However, in mixtures, the figure shows a slight but significant enhancement of placement at the low level presumably induced by the much higher level of the ground and a slight but significant suppression of the figure placement at the high level of the ground when the figure is at a lower level than the ground. It would appear that the ratio of the figure to the ground determines whether there is enhancement or suppression, but the present experiment was not designed to study this, and because of boundary effects during placement, interpretation of the results in this light is problematic. Nevertheless, at the medium level, the figure in the presence of a medium level of ground exhibited no apparent enhancement or suppression for either $\mathrm{C} 8$ or $\mathrm{C} 10$ in contrast to the results from mixtures of dissimilar smelling compounds C6 and C8 (Kurtz et al. 2010) where there was significant suppression.

Figure 4 shows the matching result when the figure was held constant and the ground was varied. Therefore, all of the figure controls were at the medium level and should produce identical placements and they did. Furthermore, the medium-level mixtures produced the same placement as all the controls, indicating no enhancement or suppression of intensity of $\mathrm{C} 8$ or $\mathrm{C} 10$ on each other at similar potencies. At the extreme levels where the figure is medium and the ground is low, there is insignificant suppression of the figure by the ground; however, at the other extreme when the ground is higher, then the figure appears as though the subjects are responding only to the ground. Unfortunately, the boundary effects experienced during placement of the figure at extreme levels complicate the interpretation of these results. High and low levels were included in the experiments to allow effects on the medium figure by the medium ground to be determined. Therefore, the medium concentration in the figures yielded the most meaningful result and was the only one used in Fig. 5. The odor matching of both octanal and decanal did not differ significantly from their controls, indicating no mixture 
suppression or enhancement. In summary, when octanal was attended to in the olfactory "analysis," it showed the same degree of enhancement as decanal did when it was the figure and the focus of attention (This allowed us to average the medium-medium results in Fig. 4 for use in Fig. 5).

\section{Discussion}

Figure 5a compares the results from the current experiment using "similar" smelling binary mixtures $\mathrm{C} 8$ and $\mathrm{C} 10$ with the results of Kurtz et al. (2009) which used the "dissimilar" smelling odorants C8 and C6. Due to the difference in the design of the two studies and boundary effect on matching at the extremes (low and high levels of stimulant), only data from the medium-level ( $8 \mathrm{M}-10 \mathrm{M}$ and $8 \mathrm{M}-6 \mathrm{M})$ samples were compared. The point at the left in Fig. 5a labeled "Similar" is the average of the $8 \mathrm{M}-10 \mathrm{M}$ data from this experiment, and the point on the right labeled "Dissimilar" is the average of the $8 \mathrm{M}-6 \mathrm{M}$ data from Kurtz et al. (2009). "Similar" and "Dissimilar" refers to the odor quality of the components in the mixture tested. The average bottle placement of the unmixed odorants is indicated by the empty triangles, of the mixed odorants by black dots and their LSI's by "whiskers.". If the unmixed bottles were matched to the $\mathrm{M}$ reference $100 \%$ of the time, the value would be 3 . The average bottle placement for unmixed bottles $8 \mathrm{M}$ and $10 \mathrm{M}$ is 2.62 , and the average bottle placement for bottles $6 \mathrm{M}$ and $8 \mathrm{M}$ is 2.97 . The average bottle placement for the "Dissimilar" binary mixtures $(6 \mathrm{M}-8 \mathrm{M})$ was significantly below that of the unmixed bottles, indicating suppression. In contrast, the bottle placement for the "Similar" binary mixture $(8 \mathrm{M}-10 \mathrm{M})$ is not significantly different from the unmixed odorants, indicating no mixture suppression between the similar smelling rORI7 agonist $\mathrm{C} 8$ and $\mathrm{C} 10$.

Figure $5 b$ compares the results from the crossadaptation studies reported in our third paper in this series, Kurtz et al. (2009), for the same "similar" and "dissimilar" smelling pairs $(8 \mathrm{M}-10 \mathrm{M}$ and $8 \mathrm{M}-6 \mathrm{M})$. The average bottle placement for the unadapted odorants is indicated by the empty triangles, and after crossadaptation, the average bottle placements are indicated by black dots and their LSIs by "whiskers." Since there was no significant difference in the results between the figure and ground presentations, they were averaged together. The "similar" smelling compounds C8 and C10 showed significant cross-adaptation, while the "dissimilar" smelling pairs C8 and C6 showed none. There seems to be a difference between mixture suppression and crossadaptation in the way odor intensity is processed.

\section{Conclusion}

In summary, results from this study (using a matching task with a reference the same as the analyte) combined with findings from Kittel et al. (2008), Kurtz et al. (2010), Kurtz et al. (2009), Goyert et al. (2007), and Frank et al. (2010) indicate a difference in the behavior between "similar smelling odorants" and "dissimilar smelling odorants" in an analytical protocol. "Analytic" mixture suppression was greater when components had different odor qualities, while odorants with similar odor qualities were less likely to suppress each other. In contrast, mixtures of odorants with similar odor qualities were more likely to show crossadaptation. Taken together, these experiments support the implications of Jinks and Laing (1999) that mixture processing is central and those of Atanasova et al. (2005) that peripheral events, e.g., differences in olfactory epithelial membrane solubility, modulate perception. The findings of the experiments reported here suggest that mixture suppression and adaptation are processed separately: perhaps one central (mixture suppression) and the other peripheral (adaptation).

\section{References}

Andrews HP, Snee RD, Sarner MH (1980) Graphical display of means. Am Stat 34(4):195-199

Atanasova B, Thomas-Danguin T, Chabanet C, Langlois D, Nicklaus S, Etievant P (2005) Perceptual interactions in odour mixtures: odour quality in binary mixtures of woody and fruity wine odorants. Chem Senses 30(3):209-217

Auvray M, Spence C (2008) The multisensory perception of flavor. Conscious Cogn 17(3):1016-1031

Berglund B, Olsson MJ (1993) Odor-intensity interaction in binary mixtures. Human perception and performance. J Exp Psychol 19 (2):302-314

Berglund B, Berglund U, Lindvall T, Svensson LT (1973) A quantitative principle of perceived intensity summation in odor mixtures. J Exp Psychol 100(1):29-38

Brossard C, Rousseau F, Dumont JP (2007) Perceptual interactions between characteristic notes smelled above aqueous solutions of odorant mixtures. Chem Senses 32(4):319-327

Cain WS, Drexler M (1974) Scope and evaluation of odor counteraction and masking. Ann N Y Acad Sci 237:427-439

Cometto-Muniz JE, Cain WS, Abraham MH, Sanchez-Moreno R (2007) Cutoff in detection of eye irritation from vapors of homologous carboxylic acids and aliphatic aldehydes. Neuroscience 145(3):1130-1137

Delwiche J (2004) The impact of perceptual interactions on perceived flavor. Food Quality and Preference 15(2):137-146

Diamond J, Breslin PAS, Doolittle N, Nagata H, Dalton P (2005) Flavor processing: perceptual and cognitive factors in multimodal integration. Chem Senses 30(suppl):i232-i233

Frank ME, Goyert HF, Hettinger TP (2010) Time and intensity factors in identification of components of odor mixtures. Chem Senses 35(9):777-787

Gottfried JA (2010) Central mechanisms of odour object perception. Nat Rev Neurosci 11(9):628-641 
Goyert HF, Frank ME, Gent JF, Hettinger TP (2007) Characteristic component odors emerge from mixtures after selective adaptation. Brain Res Bull 72(1):1-9

Jinks A, Laing DG (1999) Temporal processing reveals a mechanism for limiting the capacity of humans to analyze odor mixtures. Cogn Brain Res 8(3):311-325

Jones FN, Woskow MH (1964) On the intensity of odor mixtures. Ann N Y Acad Sci 116:484-494

Kittel KM, Kurtz AJ, Barnard J, Acree TE (2008) Free-choice profiling of OR-I7 agonists and homologues using GCO. Chemosens Percept 1(4):235-241

Köster EP (1968) Relative intensity of odour mixtures at suprathreshold level. Olfactologia, Supplements. Cahiers Olo-RhinoLaryngologie III, 1:29-41

Köster EP (1969) Intensity in mixtures of odorous substances. In: Olfaction and taste III. Rockefeller Press, New York, pp 142-149

Kurtz AJ, Lawless HT, Acree TE (2009) Reference matching of dissimilar binary odor mixtures. Chemosens Percept 4:186-194

Kurtz AJ, Lawless HT, Acree TE (2010) The cross-adaptation of green and citrus odors. Chemosens Percept 3:149-155

Laing DG, Francis GW (1989) The capacity of humans to identify odors in mixtures. Physiol Behav 46(5):809-814

Laing DG, Panhuber H, Willcox ME, Pittman EA (1984) Quality and intensity of binary odor mixtures. Physiol Behav 33:309-319

Lawless HT, Heymann H (1999) Sensory evaluation of food: principles and practices. Springer, New York
Miyazawa T, Gallagher M, Preti G, Wise PM (2009) Odor detection of mixtures of homologous carboxylic acids and coffee aroma compounds by humans. J Agric Food Chem 57(21):9895-9901

Nagata Y (2002) Measurement of odor threshold by triangle odor bag method. In: Odor measurement review. Ministry of the Environment, Japan, pp 118-127

Peterlin Z, Li Y, Ryan K, Firestein S (2008) Effect of ligand conformation on the activity of the olfactory receptor OR-I7. Chem Senses 33:S74

Prescott J (2004) Psychological processes in flavour perception. In: Taylor AJ, Roberts D (eds) Flavour preception. Blackwell, London, pp 256-278

Ramachandran VS, Hubbard EM (2001) Psychophysical investigations into the neural basis of synaesthesia. Proc R Soc Lond B Biol Sci 268(1470):979-983

Small DM, Prescott J (2005) Odor/taste integration and the perception of flavor. Exp Brain Res 166(3-4):345-357

Veldhuizen MG, Shepard TG, Wang MF, Marks LE (2010) Coactivation of gustatory and olfactory signals in flavor perception. Chem Senses 35(2):121-133

Wise PM, Cain WS (2000) Latency and accuracy of discriminations of odor quality between binary mixtures and their components. Chem Senses 25(3):247-265

Zhao H, Ivic L, Otaki JM, Hashimoto M, Mikoshiba K, Firestein S (1998) Functional expression of a mammalian odorant receptor. Science 279(5348):237-242 